\title{
Stroke Epidemiology in South, East, and South-East Asia: A Review
}

\author{
Narayanaswamy Venketasubramanian, ${ }^{\mathrm{a}}$ Byung Woo Yoon, ${ }^{\mathrm{b}}$ Jeyaraj Pandian, ${ }^{\mathrm{c}}$ Jose C. Navarro ${ }^{\mathrm{d}}$ \\ ${ }^{a}$ Raffles Neuroscience Centre, Raffles Hospital, Singapore, Singapore \\ 'Department of Neurology, Seoul National University Hospital, Seoul, Korea \\ 'Department of Neurology, Christian Medical College, Ludhiana, India \\ ${ }^{d}$ Neuroscience Institute, St. Luke's Medical Center, Jose R. Reyes Medical Center, Manila, Philippines
}

Journal of Stroke 2017;19(3):286-294

https://doi.org/10.5853/jos.2017.00234

On page 287, "The lowest rates are observed in Japan (43.4/1,000,000 person-years and Singapore (47.9/100,000 person-years), followed by Bangladesh, Papua New Guinea, and Bhutan." should be corrected as following.

\section{Corrected Sentence}

The lowest rates are observed in Japan (43.4/100,000 person-years and Singapore (47.9/100,000 person-years), followed by Bangladesh, Papua New Guinea, and Bhutan.

We apologize for any inconvenience that this may have caused. 